\title{
Transatlantica
}

Revue d'études américaines. American Studies Journal

\section{Journée d'études "Littérature et politique en Nouvelle-Angleterre"}

Paris VII, Lille III, ENS, 31 janvier 2009, ENS Ulm

\section{Thomas Constantinesco et Antoine Traisnel}

\section{(2) OpenEdition}

\section{Journals}

\section{Édition électronique}

URL : https://journals.openedition.org/transatlantica/4335

DOI : 10.4000/transatlantica.4335

ISSN : 1765-2766

\section{Éditeur}

Association française d'Etudes Américaines (AFEA)

\section{Référence électronique}

Thomas Constantinesco et Antoine Traisnel, « Journée d'études "Littérature et politique en NouvelleAngleterre" », Transatlantica [En ligne], 1 | 2009, mis en ligne le 02 juillet 2009, consulté le 18 septembre 2021. URL : http://journals.openedition.org/transatlantica/4335 ; DOI : https://doi.org/10.4000/ transatlantica. 4335

Ce document a été généré automatiquement le 18 septembre 2021.

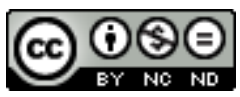

Transatlantica - Revue d'études américaines est mise à disposition selon les termes de la licence Creative Commons Attribution - Pas d'Utilisation Commerciale - Pas de Modification 4.0 International. 


\section{Journée d'études "Littérature et politique en Nouvelle-Angleterre"}

Paris VII, Lille III, ENS, 31 janvier 2009, ENS Ulm

Thomas Constantinesco et Antoine Traisnel

1 Précédée de sa lettre, l'Amérique a d'emblée partie liée avec la littérature et s'origine dans la différence d'avec son écriture : qu'il s'agisse de la "Plantation du Seigneur ", établie pour accomplir la prophétie des Écritures, ou de l'Amérique contractuelle que produit la Constitution, elle est le fruit imaginaire d'une écriture avant même que ne lui corresponde un territoire. Mais elle est aussi cette Nouvelle-Angleterre, en rupture de ban avec la Mère Patrie, qui s'imagine autre et s'efforce de conjurer le spectre d'un Vieux Monde qu'elle ne cesse pourtant de convoquer pour s'y comparer. Longtemps, la Nouvelle-Angleterre fut le trope de l'Amérique tout entière ; elle en tenait lieu. Et c'est par les Lettres que la Nouvelle-Angleterre a cherché à incarner l'Amérique, à la contenir en ses frontières, de sorte que sa littérature semble d'essence politique, au sens étymologique du terme, car elle vise et engage tout à la fois le devenir de la communauté.

2 Les participants à cette journée d'études étaient ainsi invités à s'intéresser à ce que la Nouvelle-Angleterre, dans ses textes, dit du projet politique de l'Amérique, au double sens du génitif. Il s'agissait de s'interroger sur ce que Jacques Rancière appelle «la politique de la littérature ", moins pour déceler des références au contexte politique d'une période historique donnée, que pour envisager la manière dont, en NouvelleAngleterre, «la littérature fait de la politique en tant que littérature ${ }^{1}$ ». Dans quelle mesure la littérature de Nouvelle-Angleterre participe-t-elle à l'élaboration du corps politique de l'Amérique, alors en perpétuelle mutation? De quelle manière cette communauté, soutenue par la rhétorique normative du e pluribus unum, incorpore-t-elle les individus qui la composent? La "littérature », comme production spécifique et historiquement marquée, induit un rapport inédit au monde et opère un " partage du sensible ", pour citer à nouveau Jacques Rancière ${ }^{2}$, propositions que cette journée d'études entendait confronter au cas particulier des écrits de Nouvelle-Angleterre. Fiction à la fois politique et littéraire, la Nouvelle-Angleterre s'écrit d'abord comme une 
«Cité sur la colline » que la littérature puritaine invente sous la forme d'un avant-poste de la "Réformation ». Deux siècles plus tard, c'est encore sous les espèces de la fiction qu'elle entend être la figure de l'Amérique, alors que le centre de gravité de la nation s'est déplacé vers le Sud et l'Ouest, à la suite des bouleversements générés par l'expansion territoriale, la révolution industrielle, la démocratie jacksonienne et la Guerre Civile. C'est ce moment qui a intéressé la plupart des communications de la journée, moment paradoxalement baptisé près d'un siècle plus tard la « Renaissance américaine ", comme si la Nouvelle-Angleterre ne pouvait jamais tout à fait se défaire de la référence à la Vieille Europe. Mais avant d'en arriver au XIX ${ }^{e}$ siècle et à plusieurs de ses figures emblématiques, la journée s'est ouverte sur un retour en arrière vers la préhistoire puritaine.

3 New England: c'est à l'endroit de l'espace typographique qui indissociablement césure et jointure la « Nouvelle Angleterre » que Cécile Roudeau (Paris III) propose de placer sa communication. Cette espace fait de la Nouvelle-Angleterre le lieu de l'écart et la destine à la récriture, qu'elle soit traduction des vieilles lettres européennes ou commentaire de la Lettre biblique. La lettre en Nouvelle-Angleterre est "toujours désajustée ", elle se fissure et fonde le commun sur le "partage du sensible » dont parle Rancière. Cécile Roudeau entend mettre cette hypothèse à l'épreuve du récit de captivité de Mary Rowlandson. Dans les transactions qui s'opèrent entre la Lettre biblique et la "voix indienne" se dessinent dans ce texte les frontières de la communauté. Si la Lettre des Écritures, que Mary porte à travers la wilderness, sauve et protège, elle tient aussi la narration captive et tente d'interdire une intimité autre - un désir de goûter à cet autre de la Lettre, qui captive et attise les sens. Le texte par moments s'ensauvage, sans présenter toutefois un mouvement progressif vers l'indianisation. Si le retour à l'enclos est programmé, sans doute désiré, "home » devient un signifié flottant, venant tantôt désigner la plantation, tantôt le campement indien. La narration se clôt alors sur un écart, projeté au coeur même de la lettre (home, we). Plutôt que de fissurer les assises de la communauté, cet écart, qui ne saurait figurer sur aucune carte, assure le fondement paradoxal d'un « nous » américain. C'est dès lors en tant que lettre que l'écart devient politique en Nouvelle-Angleterre, lettre minuscule qui s'ouvre et donne lieu au lieu sans attaches de la différence interne, lieu poétique et politique, lieu de production (performance) d'un « en commun ».

Les travaux de la journée se sont poursuivis avec trois communications consacrées à Thoreau. Hélène Thiercy (Paris VII) s'est d'abord intéressée au premier et unique numéro des Aesthetic Papers, publié par Elizabeth Peabody en 1849, et a notamment proposé une lecture comparée de deux des essais qu'il contient: "Abuse of Representative Government », de S. H. Perkins, et "Resistance to Civil Government ", de Thoreau. Si ces textes portent tous deux sur la nature du pouvoir politique dans le contexte de la guerre du Mexique et du débat sur l'esclavage, ils articulent des conceptions antithétiques du rapport entre les citoyens et le gouvernement. Le texte de Perkins s'inscrit dans la tradition des Federalist Papers et prône une conception républicaine du gouvernement analogue à celle de Hamilton, où les représentants, élus pour leurs qualités supérieures et sourds aux pressions des masses ignorantes, ont pour tâche de mettre en œuvre des idéaux de justice et d'équité qui doivent certes permettre d'éduquer le peuple et de réformer la communauté, mais qui, du même coup, légitiment un pouvoir fort. Or c'est précisément cette mécanique du pouvoir que dénonce Thoreau : appelant tout citoyen à résister à un gouvernement qu'il juge illégitime, il met en scène le récit de sa propre désobéissance et recentre le débat sur l'individu, qui 
ne doit s'en remettre qu'à lui-même. L'écrivain devient ainsi le héraut, mais aussi le héros, de cette entreprise de réforme individuelle, nécessaire à toute refondation sociale. L'union de soi à soi, au plus profond de l'intimité, prend alors des allures de déclaration de guerre: "I quietly declare war with the State, after my fashion ", annonce Thoreau vers la fin du texte. À sa façon, le sujet refuse toute forme d'assujettissement et proclame son indépendance, semblant faire de la résistance la vertu civique par excellence.

5 Reprenant cette idée pour mieux la faire vaciller, Michel Imbert (Paris VII) s'est interrogé sur les deux titres sous lesquels on connaît le texte de Thoreau : «Resistance to Civil Government » et «Civil Disobedience ». De l'un à l'autre, l'adjectif «civil» change de place et qualifie alternativement la puissance publique et la dissidence privée. Pour brouiller un peu plus les cartes, l'acte même de désobéissance, dont le récit constitue le cœur de «Civil Disobedience » et qui valut à Thoreau un bref séjour dans la prison de Concord, fait l'objet de témoignages autobiographiques dans A Week on the Concord and Merrimack Rivers et Walden. Bien que publiés à plusieurs années d'intervalle, ces trois textes sont contemporains, de sorte que la question de la résistance paraît relever tout aussi bien du pamphlet politique que des écrits intimes. "Civil Disobedience " peut en effet se lire comme un récit de captivité où l'on devine, en filigrane, tout un roman familial: l'État corrompu y est féminisé, tandis que la résistance, axiome préalable de la démocratie, est décrite comme la marque même de la virilité. La régénération de l'État passe alors par la renaissance d'un homme nouveau et la cellule dans laquelle il est enfermé devient une matrice de substitution où le citoyen résistant s'enfante lui-même en homme libre en attendant de pouvoir transférer la cité des hommes en pleine nature. Mais le retour à l'État de nature qu'imaginent les textes « écologiques » de Thoreau s'avère moins triomphal que prévu. Lorsque, dans Walden, il décrit l'érosion du talus à la fonte des neiges ou lorsque, dans The Maine Woods, il retrace l'ascension du mont Ktaadn, les métaphores qui prolifèrent dans le texte sont de part en part politiques et viennent remettre en cause l'idéal de refondation de la société selon un modèle pré-pastoral. Se pensant le dépositaire de la loi naturelle, le sujet est en réalité déposé et se découvre assujetti aux forces irrésistibles de la nature qui sont aussi celles de l'écriture. Il ne domine ni la nature, ni les lettres, car elles sont gouvernées par des lois propres. Alors, peut-être, faut-il aussi entendre les appels répétés à la résistance au sens psychanalytique du mot, comme la résistance du sujet à l'aveu de sa condition de sujet, comme le déni de son propre dessaisissement.

Danielle Follett (Paris VIII) s'est également penchée sur les liaisons dangereuses que le sujet entretient avec la nature. Elle emprunte le titre de sa communication, « Give me a culture that imports muck from the meadows ", à la conférence « Walking » donnée par Thoreau en 1851. Cette phrase est l'occasion pour Danielle Follett de réfléchir à la question du rapport que la jeune culture américaine devrait, selon Thoreau, entretenir avec la nature, du moins un certain type de nature. En effet, la nature dont parle Thoreau n'est pas celle des jardins bien enclos, mais celle, sauvage, des grands espaces, la wilderness, ou plutôt la wildness, c'est-à-dire une nature qui s'offre comme totalement vierge ou intouchée. Les plantes en décomposition, le lisier, la vase et la boue sont envisagés comme les éléments essentiels à la constitution d'un esprit sain, et plus généralement d'une écriture et d'une culture saines: si l'écrivain ne marchait pas quotidiennement à travers cette nature, son écriture serait anémique et débile. À l'écrivain incombe alors la tâche d'importer la boue depuis les terres incultes jusqu'à la ville, à lui de prélever l'engrais nécessaire à la civilisation lors de ses excursions dans 
l'Ouest sauvage. Étonnamment, cet impératif entre en résonance avec la doctrine millénariste de la Destinée Manifeste : "Westward the star of empire takes its way ». Thoreau reformule ainsi le paradoxe de la Frontière, qui voit le Pionnier en quête de territoires sauvages y semer fatalement les graines de la civilisation et de fait épuiser la nature à mesure qu'il la conquiert, et fait de la nature le lieu où se régénère l'Amérique.

7 Les dernières communications de la journée ont été consacrées à trois autres figures « représentatives » de la Nouvelle-Angleterre et de ses Lettres: Emerson, Hawthorne et James. Poursuivant la réflexion initiée par Michel Imbert sur le mélange des genres, Thomas Constantinesco (Paris VII) s'est attaché à lire les essais d'Emerson comme cette fiction qui mettrait en scène les aventures d'un héros représentatif. Malgré l'aversion qu'il éprouve habituellement pour la fiction narrative, Emerson voit dans ce qu'il appelle "the romance of character" le lieu exemplaire où se représente un modèle de vertu citoyenne que le poète incarne mieux que tout autre. Le texte dresse toutefois un portrait contradictoire du grand homme de lettres. Il est d'une part envisagé comme le représentant d'un corps électoral divisé qu'il semble seul capable de remembrer en une communauté de libres égaux. Mais d'autre part, ses pouvoirs poétiques légitiment sa distinction sociale : il revendique le privilège de sa force de caractère pour s'arroger tous les pouvoirs et contenir l'Amérique dans l'orbe de son «moi impérial». Toutefois, comme pour prévenir le risque de dérive autocratique, un système de "rotations", orchestré par la nature, vient garantir la succession impersonnelle des représentants à la tête de l'État, empêchant la concentration du pouvoir entre les mains d'un seul homme. Le texte naturalise l'alternance politique et chaque citoyen est cité à comparaître publiquement devant ses pairs. Cette comparution s'apparente à une scène romanesque de reconnaissance mutuelle qui se mue en un exercice de scrutation du visage de l'autre, de déchiffrement d'un corps qui se fait texte. Or les corps lisibles sont aussi corps scriptibles, car le caractère individuel et national s'inscrit à même le corps. Les essais deviennent alors littéralement la romance d'un caractère dont le héros serait peut-être la lettre « 0 ». C'est en effet autour de cette lettre-pictogramme que s'organise l'imaginaire politique du texte, tendu entre le cercle de la communauté démocratique et l'enclos d'un « moi » (I) dont l'œil (eye) englobe le monde. Mais loin de résorber cette tension, la déclinaison de figures circulaires au fil du texte ne se laisse jamais ramener à une formule univoque. Les figures représentatives se figurent entre elles sans qu'il soit possible d'en revenir à un sens supposé propre, engageant le lecteur à parcourir les intervalles qui les relient en les séparant. Les essais peuvent alors être lus comme le lieu de cet écart où cherche à s'inventer l'être-ensemble et c'est aussi en cela qu'ils sont la romance d'un caractère, style « Nouvelle-Angleterre ».

8 C'est également au statut politique de la romance que s'est attaché Antoine Traisnel (Lille III / Paris VIII), à partir d'une étude de The Blithedale Romance (1852). L'ouvrage s'est en effet souvent vu reprocher son manque de moralité, moins à cause de prises de positions scandaleuses à l'égard de l'esclavage ou de l'émancipation des femmes qu'en raison de son manque de lisibilité politique. Nombre de critiques se sont donné pour mission de racheter la romance de Hawthorne en lui attribuant une morale, un supplément de texte censé donner un sens explicite au récit. Partant de l'analyse d'un article de Michael Colacurcio, Antoine Traisnel propose d'examiner le geste critique qui consiste à moraliser un texte pour en extraire la teneur politique. La morale requiert en effet l'identification d'une autorité extérieure au texte, soit d'un auteur, entendu comme principe de cohérence et origine d'un « dire »; or, et c'est en ceci que le livre 
est amoral, l'auteur de The Blithedale Romance semble s'être retiré de son texte. L'« Auteur" de la préface professe ne vouloir énoncer aucune théorie à l'égard des questions de société abordées de façon oblique par la romance. Paradoxalement, en se dédisant, cette profession de foi réaffirme la présence d'un "dire». Empruntant à l'analyse de Rancière, qui renverse les polarités traditionnelles du «dire" et du «faire » en faisant du premier le propre du politique et du second le propre de la littérature, Antoine Traisnel suggère que le texte de Hawthorne défait le processus de subjectivation tout en anticipant avec ironie sa récupération politique, opposant à la morale de l'histoire une éthique de l'indétermination inhérente à l'écriture.

La journée s'est achevée avec l'exposé d'Agnès Derail-Imbert (ENS Ulm / Paris IV), consacré aux Bostonians de Henry James. Publié en 1886, le roman se donne comme un "conte» social et politique de Nouvelle-Angleterre, s'inscrivant de fait dans la tradition inaugurée par Hawthorne. C'est du reste peut-être ce qui lui valut de ne pas figurer dans l'Édition de New York. Trop réaliste et trop local, il n'avait pas sa place dans " la Maison de la Fiction ", à moins que James n'ait choisi d'émanciper l'œuvre de la tutelle auctoriale pour la mettre en circulation dans l'espace public. Interrogeant la place de la parole privée dans la sphère publique, c'est-à-dire aussi bien de l'intimité en démocratie, The Bostonians a pour thème le suffrage des femmes à l'heure de la Reconstruction. La Guerre Civile se poursuit dans le roman sous la forme d'une guerre des sexes qui est aussi un conflit sectionnel et la scène sociale qu'il dépeint est perçue depuis l'intériorité des consciences privées, en particulier celles, antagonistes, de Basil Ransom, séduisant sudiste désargenté, et de sa cousine, Olive Chancellor, riche vieille fille de Boston. Tous deux s'affrontent pour la possession de Verena Tarrant, dont Olive veut faire le porte-parole de la cause des femmes, tandis que Basil cherche à en faire son épouse. Verena, en tant précisément qu'elle est dépourvue d'intériorité, sert de médium impersonnel où sont mis en scène et produits les désirs intimes d'Olive et de Basil, de sorte que l'intime démocratique ne semble accessible que comme spectacle. Mais en se produisant sur scène, Verena finit par «se " produire : elle se crée comme un soi, comme un sujet où s'ouvre une intériorité dans laquelle elle diffère du rôle que les autres personnages voudraient lui voir jouer. Pour autant, ce soi ne s'appartient pas en propre et sera bientôt ravi par Ransom. Au terme du roman, l'union conjugale de Ransom et de Verena, désirée mais non choisie par cette dernière, porte la division au plus intime du sujet démocratique qui ne se découvre libre que de ne pas l'être. Mais si l'épilogue vient interrompre le processus d'épanouissement de la conscience, le roman en aura malgré tout fait le récit. La croissance du roman est en effet coextensive à l'expansion de la conscience de Verena qui, à ce titre, est une figure de l'œuvre. La narration suit l'ouverture de sa vision: son regard crée le monde qu'il regarde et, d'actrice, elle devient spectatrice. Sa part intime se manifeste ainsi comme un mouvement vers le dehors médiatisé par le filtre de la conscience. L'intime serait alors le mouvement de cette sortie, communiqué à la communauté des lecteurs. En écartant The Bostonians de l'Édition de New York, James prolongerait le geste de l'étrange narration de son roman : confiant le texte sans préface, ni contrôle d'auteur, à la scène démocratique, il s'abstiendrait de faire valoir sa prérogative privée, comme si le privilège de l'intime n'était que celui de l'œuvre.

10 Nous ne saurions restituer ici, ni la richesse, ni la variété des échanges qu'ont suscités ces communications et auxquels a contribué un public nombreux. Une problématique d'ensemble s'est néanmoins fait jour au fil des interventions et des discussions : si, en Nouvelle-Angleterre, la littérature est d'abord le lieu où cherche à s'inscrire et 
s'énoncer la lettre de la Loi (religieuse, politique, sociale, sexuelle), il semble bien souvent que les Lettres de Nouvelle-Angleterre obéissent à des lois qui leur sont propres. La lettre y entre en dissidence avec la Loi et ce dissensus apparaît comme le fond sans fond de la communauté qu'imaginent les fictions de Nouvelle-Angleterre.

\section{NOTES}

1. Jacques Rancière, Politique de la littérature, Paris, Galilée, 2007, p. 11

2. Jacques Rancière, Le Partage du sensible. Esthétique et politique, Paris, La Fabrique, 2000.

INDEX

Thèmes : Actualité de la recherche

\section{AUTEURS}

THOMAS CONSTANTINESCO

\section{ANTOINE TRAISNEL}

Université Paris VII - Denis DiderotUniversité Charles de Gaulle - Lille III 